\title{
Chemical-shift MRI for diagnosis thymoma in myasthenia gravis: Value of qualitative and quantitative assessment
}

Huynh Quang Huy ${ }^{1 *}$ and Phung Anh Tuan ${ }^{2}$

*Correspondence: huyhq@pnt.edu.vn

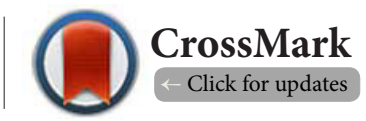

${ }^{1}$ Radiology Department, Pham Ngoc Thach University of Medicine, Vietnam. ${ }^{2}$ Imaging Diagnostic Center, Vietnam Military Medical University, Vietnam.

\begin{abstract}
Objectives: To evaluate the usefulness of chemical-shift magnetic resonance imaging (MRI) in patients with myasthenia gravis (MG) for differentiating thymoma from normal thymus and thymic hyperplasia.

Patients and Methods: 62 patients with MG were treated surgically in 103 Military Hospital and Cho Ray Hospitals from August 2014 to January 2017. All patients had undergone surgery to remove thymus, thymoma, and had pathology reports. All of them had not received any neoadjuvant chemo-radiotherapeutic treatment. MRI examination was obtained using a 1.5 T MRI unit (Intera, Philips Healthcare, the Netherlands) from the thoracic inlet to the cardiophrenic angle. Comparison of the chemical shift ratio(CSR) between two groups was processed by the T-Student test. Logistic multivariate regression was performed to estimate the probability that patients had thymoma by both predictive qualitative and quantitative variables. A $\mathrm{p}$ value of $<0.05$ was considered indicative a statistically significant differencefor all statistical analyses.

Results: 62 patients included 24 males (45.2\%), 38 females (54.8\%). Comparision with pathologic results, the sensitivity, the specificity and the accuracy of qualitative assessment were $97.3 \%, 88 \%, 93.5 \%$, respectively. CSR value was very good criteria for distinguishing between thymoma and non-thymoma groups with the AUROC of 0.984 (95\% CI: 0.914-1.000), and with an optimal cut-off point of 0.825. Applying this cut-off point, the specificity and the accuracy of quantitative assessment were 100\%, 96\%, 98.4\%, respectively. An increase in 0.1 value of CSR for all shape, location and signal intensity leads to an increase of 10 times in the probability of finding thymoma.

Conclusion: Chemical shift magnetic resonance imaging is helpful in differentiating thymoma from normal or hyperplastic thymus based on quantifying fat tissue.

Keywords: Chemical-shift MRI, thymoma, myasthenia gravis

Myasthenia gravis (MG) is the most common autoimmune disease. The thymus plays a central role in MG pathophysiology mechanism. Approximately $90 \%$ MG patients displayed thymic abnormalities, such as hyperplasia (70\%) and thymoma (20\%) [1]. The discrimination thymoma from thymic hyperplasia is really important, especially in surgical treatment. Thymectomy is strongly recommended in all thymoma cases, whereasthe surgical indication in hyperplasia cases should be only considered with less effective conservation treatments. The discrimination is based on morphological assessments at CT. Thymoma is a

focal soft tissue mass. Thymic hyperplasia displays a diffuse symmetric enlargement gland. However, many studies have also shown that it is difficult to differentiate TLH from thymoma on CT. TLH may display as a focal soft mass or on the contrast thymoma has diffuse enlargement form in both lobes. In this cases, chemical-shift MRI could be helpful for differentiation based on detecting fat in tissue.

The aim of this study was to evaluate the usefulness of chemicalshift magnetic resonance imaging (MRI) in patients with myasthenia gravis (MG) for differentiating thymoma from normal thymus orthymic hyperplasia.
\end{abstract}

\section{Introduction}


Huy et al, Medical Imaging and Radiology 2019,

\section{Patients and methods Patients}

62 patients with MG were treated surgically in 103 Miliraty Hospital and Cho Ray Hospitals from August 2014 to January 2017. The inclusion criteria: Patients over 16 years old were definitely diagnosed MG. All patients had undergone surgery to remove thymus, thymoma, and had pathology reports. All of them had not been received any neoadjuvant chemo-radiotherapeutic treatment.

\section{MRI protocol}

MRI examination was obtained using a 1.5 T MRI unit (Intera, Philips Healthcare, the Netherlands) from the thoracic inlet to the cardiophrenic angle. All patients underwent transverse gradient-echo T1-weighted in and opposed-phase imaging, using an anterior-to-posterior phase-encoding direction. In 57 patients, dual-echo technique in single breath hold was performed. In 5 patients non dual-echo technique in separated breath holds was performed. Imaging parameters included $350 \mathrm{~mm}$ field of view, 256×256 image matrix, $5 \mathrm{~mm}$ section thickness, $151 \mathrm{~ms}$ time repetition (TR), and in-phase and opposed-phase time echo (TE) of 4.6 and $2.3 \mathrm{~ms}$. Axial T1-weighted, T2-weighted and sagittal T2-weighted with fat suppression, black blood technique with cardiac gate were added. Imaging parameters included TR 1000, 2000ms, TE 10, $60 \mathrm{~ms}$ and section thickness of $6 \mathrm{~mm}$.

\section{Image analysis}

Two radiologists with 20 and 23 years' experience and without knowledge of patients' information independently analyzed for qualitative and quantitative imaging data.

The signal intensity measurements within the thymus and chest wallmuscle were obtained by using region-of-interest (ROI) (area, $0.5-1 \mathrm{~cm}^{2}$ for the thymusgland, and $1-2 \mathrm{~cm}^{2}$ for the chest wall muscle, respectively). The selection of the ROI placement should be first made on the opposed-phase image, on the area which exhibits the highest signal intensity and then mirrored on the in-phase image in the exact same position and same size. Measure in the central position, not peripheral areas to avoid partial volume effects. Avoid the areas of void signal at the interfaces between the fat-dominant and waterdominant tissues (India ink artifact). Avoid cystic or necrotic components on the T2-weighted images with and without fat-suppressed. On the chest wall muscle, avoid fat strips.

\section{Criteria for evaluating image parameters}

The gland shape divided into two forms: diffuse enlargement gland (triangle, arrow) and soft tissue mass. The gland signal intensity on T1-weighted and T2-weighted was divided into three levels as lower or equal to muscle, higher than muscle but lower than fat and equal to or greater than fat. Mass shape was divided into three forms: round, oval and plaque based on the ratio of the long axis and short axis. If it was less than 1.5 , between 1.5 and 3 , and equal or greater than 3 , the tumor was considered to be round, oval, and plaque shaped, respectively [2]. The size of tumor was measured in three dimensions. The longest axis was measured at the level where the tumor appeared largest on the cross-sectional image. The short axis was measured perpendicularly longest axis at the same image [3]. The mass edge was divided into three types as smooth, lobulated and irregular [2]. Smooth lesions are typically spherical or ovoid in shape. A lobulated contour is one that exhibits one or more lobulations, characterized as convex tumor contours with adjacentnotches between tumor lobules [4]. The mass capsule was divided into three types as almost complete, partial and no capsule based on the visibility of a low-intensity thin-rim in the periphery of the tumor. If it was more than two-thirds, or less thantwo-thirds of the perimeter of tumor, the capsule was considered to be almost complete or partial, respectively [3].

\section{Criteria for thymoma on MRI}

Based on the obtained image parameters, the team made assumptions that patients have thymoma or not. Pirronti T showed the morphological criteria on $\mathrm{CT}$ to differentiate thymoma from hyperplasia [5]. On MRI, in addition to the same morphological criteria used for CT, Priola AM showed the specific MRI criteria for differentiation based on the absence of signal intensity loss on the opposed-phase relative to the in-phase image [6].

\section{Statistical analysis}

To assess the suitable level between the two researchers for each MRI parameter. Qualitative and quantitative parameters were processed by Kappa and ICC, respectively. Comparisonof qualitative imaging characteristics in two groups of thymoma and non-thymoma were processed by Chi-Square test and Fisher's exact test. Comparison of the chemical shift ratio(CSR) between two groups was processed by the T-Student test. Logistic multivariate regression was performedto estimate the probability that patients had thymoma by both predictive qualitative and quantitative variables. To evaluate values of MRI by comparison with histopathological results based on the $2 \times 2$ matrix. Se, Sp, Acc of MRI using qualitative parameters were defined. To use CSR value as the sole criteria for thymoma diagnosis, draw the ROC chart, calculate AUROC, determine the optimum cut-off point by the Youden index. Define Se, $\mathrm{Sp}$ and Acc of MRI when using this cut-off point. To combine both analyses, misdiagnosis cases on qualitative analysis were rediagnosed based on determination of CSR threshold value. Define Se, Sp, Acc of MRI using both parameters to distinguish. A $p$ value of $<0.05$ was considered indicative a statistically significant difference for all statistical analyses.

\section{Results}

Among 62 patients including 24 males (45.2\%), 38 females $(54.8 \%)$, the ratio of men and women was 1:1,2. Age of men $40.8 \pm 11.8$, women $43 \pm 14.3, p=0.514$. Histopathological lesions 
Huy et al, Medical Imaging and Radiology 2019,

included 37 thymoma, 21 hyperplasia, 4 normal thymus glands. Age of thymoma group $47.9 \pm 10.7$ years old, non thymoma group $33.2 \pm 11.6$ years old; $p<0.0001$. There were 20 males and 17 females with thymoma, $p=0.087$.

The signs distinguishing between thymoma and not thymoma are listed in Table 1. Results of Table 1 showed the qualitative signs of location, position, shapes, signal intensity loss on the opposed-phase image relative to the in-phase image, cyst, necrosis, signal intensity on T2W fat sat having significant difference between thymoma and non thymoma groups. After qualitative analysis with MRI, readers assigned a presumptive diagnosis of thymoma or not based on the definitive criteria. Comparision with pathologic results, Table 3 showed the sensitivity, the specificity and the accuracy of qualitative assessment were $97.3 \%, 88 \%, 93.5 \%$, respectively.

Quantitative assessment was report in the Figures 1 and 2. In the Figure 1, the mean CSR in the thymoma group was higher than that in the non thymoma group, $1.02 \pm 0.07$, compared with $0.64 \pm 0.1$. The difference was statistically significant $(p<0.0001)$. In Figure 2 , The AUROC of CSR in discriminating between thymoma and non-thymoma groups was 0.984 (95\% $\mathrm{Cl}: 0.914-1.000$ ) with an optimal cut-off point of 0.825 (Youden Index J=0.96). Applying this cut-off point, Table 3 showed the sensitivity, the specificity and the accuracy of quantitative

Table 1. Qualitative features of thymoma and non-thymoma groups on MRI.

\begin{tabular}{lllll}
\hline Feature n=62 & & Thy (37) & Non-thy (25) & p \\
\hline Location & Superior & $19(51.4)$ & $23(92)$ & $0.001^{\text {a }}$ \\
& Inferior & $18(48.6)$ & $2(8)$ & \\
\hline Position & Middle & $10(27)$ & $17(68)$ & $0.001^{\mathrm{a}}$ \\
& Right, left & $27(73)$ & $8(32)$ & \\
\hline Shape & Gland & $3(8.1)$ & $19(76)$ & $<0.0001^{\mathrm{a}}$ \\
& Mass & $34(91.9)$ & $6(24)$ & \\
\hline Signal intensity loss & Yes & $1(2.7)$ & $22(88)$ & $<0.0001^{\mathrm{a}}$ \\
& No & $36(97.3)$ & $3(12)$ & \\
\hline Necrosis, cyst & Yes & $17(45.9)$ & $0(0)$ & $<0.0001^{\mathrm{a}}$ \\
& No & $20(54.1)$ & $25(100)$ & \\
\hline Signal intensity T1 & $>$ SI of mus, $<$ SI of fat & $34(91.9)$ & $22(88)$ & $0.678^{\mathrm{b}}$ \\
& Equal to SI of fat & $3(8.1)$ & $3(12)$ & \\
\hline Signal intensity T2 & $>$ SI of mus, $<$ SI of fat & $35(94.6)$ & $21(84)$ & $0.210^{\mathrm{b}}$ \\
& Equal to SI of fat & $2(5.4)$ & $4(16)$ & \\
\hline Signal intensity T2 fatsat & High & $36(97.3)$ & $10(40)$ & $<0.0001^{\mathrm{b}}$ \\
& Low & $1(2.7)$ & $15(60)$ & \\
\hline
\end{tabular}

a. Chi-Square test, Asymp. Sig. (2-side);b. Fisher's test, Exact Sig. (2-side)

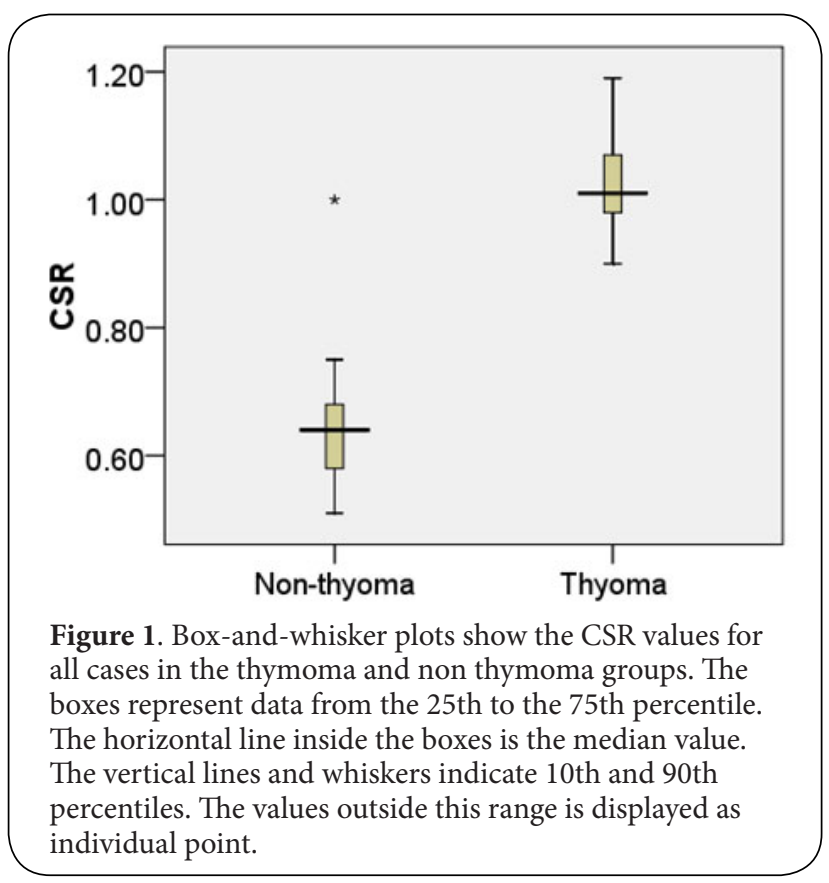

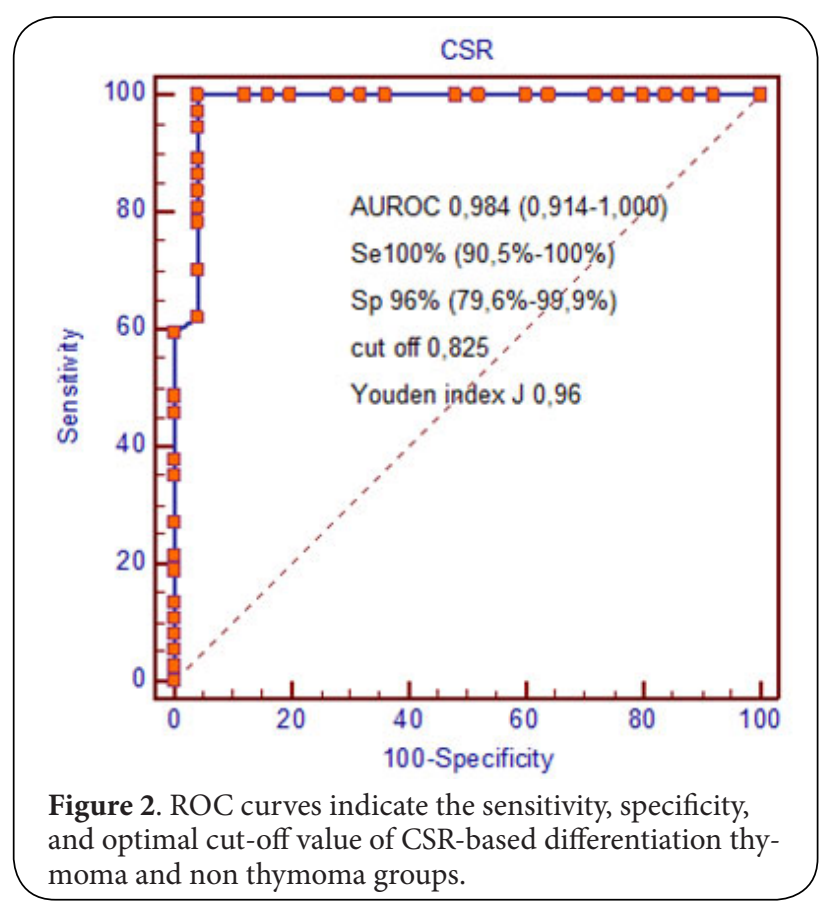


Huy et al, Medical Imaging and Radiology 2019,

http://www.hoajonline.com/journals/pdf/2054-1945-7-2.pdf

doi: $10.7243 / 2054-1945-7-2$

Table 2. Logistic regression models for evaluation of the ability of CSR to discrimination thymoma from non-thymoma.

\begin{tabular}{llll}
\hline Feature & OR & 95\%CI (lower-uper) & $\mathbf{p}$ \\
\hline Shape (gland-mass) & 29.311 & $0.124-6917$ & 0.226 \\
\hline Location (superior-inferior) & 15.032 & - & 0.633 \\
\hline Position (middle-right, left) & 0.780 & $0.002-387.707$ & 0.938 \\
\hline Necrosis, cyst (no-yes) & - & - & 0.998 \\
\hline SI T2 fatsat (low-high) & - & - & 0.999 \\
\hline SI loss (yes-no) & - & - & 0.999 \\
\hline CSR (per 0.1) & 10.566 & $1.686-66.229$ & 0.012 \\
\hline
\end{tabular}

Table 3. Ability of qualitative, quantitative and both analyses for MRI in differentiating thymoma and non-thymoma groups.

\begin{tabular}{llll}
\hline MRI & Se: \% (n) & Sp: \% (n) & Acc: \% (n) \\
\hline Qualitative & $97.3(36 / 37)$ & $88(22 / 25)$ & $93.5(58 / 62)$ \\
\hline Quantitative & $100(37 / 37)$ & $96(24 / 25)$ & $98.4(61 / 62)$ \\
\hline Both & $100(37 / 37)$ & $96(24 / 25)$ & $98.4(61 / 62)$ \\
\hline
\end{tabular}

assessment were $100 \%, 96 \%, 98.4 \%$, respectively. Using this cut-off point to rediagnose cases with erroneous presumptive diagnosis at qualitative assessment, the sensitivity, the specificity and the accuracy of both analyses were $100 \%, 96 \%$, $98.4 \%$, respectively. This results were similar as the result of alone quantitative assessment.

Although Fisher's exact test and T-Student test (Table 1, Figure 1) analysis demonstrates statistically significant differences among groups for most of the qualitative parameters as well as CSR, at logistic multivariate regressions, only CSR was significant for discrimination between groups (OR per 0.1 increase, 10.566; 95\% Cl: 1.686-66.229; Table 2). Therefore, an increase in 0.1 value of CSR for all shape, location and signal intensity leads to anincrease of 10 times in the probability of finding thymoma.

\section{Discussion}

Signs distinguish between thymoma and not thymoma The thymic hyperplasia located at the anatomic location of the gland in the upper anterior mediastinum, just in the midline. Thymoma can be encountered in any location. According to Marom EM, although the majority of thymomas located in the upper anterior mediastinum, thymoma may be still found in other sites [7]. Nasseri F commented that about half of thymoma located in the upper mediastinum and a half in the lower one [8]. Horizontally, the authors also found that thymoma rarely located in the midline. McErlean $A$ evaluated the majority of thymoma located either left or right diversion [9]. The studies of Jeong YJ, Jung KJ showed that thymoma located in the midline only accounted for below $25 \%[10,11]$.

Baron RL determined that the thymus may have the normal shape of the gland (triangle, arrow, two lobes) or a focal mass shape [12]. Popa $G$ found that the focal mass shape on CT accounted for $81.16 \%$ of 69 thymoma cases, $14.02 \%$ of the
271 hyperplasia cases and 3\% of the normal thymus cases [13]. Priola AM's study found that the mass form was found in 20/22 thymoma cases, and in 12/65 hyperplasia cases [6]. In our study, up to $34 / 37$ (91.9\%) thymoma were in mass form. Conversely, only 6 out of 25 not thymoma cases (24\%) were in mass form. Focal mass form is an important feature that distinguishes between thymoma and non thymoma.

In our study, the majority of cases had a heterogeneous signal and had a higher intensity than the muscle, smaller than the fat on both T1-weighted and T2-weighted. The signal intensity did not make significant in distinguishing between thymoma and non-thymoma. According to Takahashi K, normal or abnormal thymus should not be evaluated solely on MRI signal intensity because these characteristics varied considerably with age [14]. Marom EM also found that the signal characteristics on T1-weighted, T2-weighted of the thymoma were nonspecific and indistinguishable from normal thymus [7]. Conversely, the T2-weighted fat sat was valuable to distinguish between thymoma and non-thymoma. Normal or hyperplastic thymus deposited great fat so that the gland usually had low signal on STIR. Thymoma which without fat should have high signal. In our study, up to $36 / 37$ thymoma cases had high signal on STIR whereas only 15/25 non-thymoma cases showed high signal.

In-phase and opposed-phase sequences are very sensitive in detecting tiny fat. There was signal intensity loss on the opposed-phase image relative to the in-phase image for tissue consisting on both fat and water. Thymoma did not have fat while thymic hyperplasia occurred great fat deposition in adult. Thus, the normal or hyperplastic thymus showed the signal intensity loss but the thymoma did not. Studies of Inaoka T [15], Popa G [16], Priola AM [6] showed that signal intensity loss on the opposed-phase image is important for distinguishing between thymoma and non-thymoma. The results in Table 1 showed up to 36/37 thymoma cases did notexhibit the signal intensity loss, whereas only $3 / 25$ non thymoma cases did not $(p<0.0001)$ (Figures 3 and 4$)$.

\section{CSR value}

Figure 1 showed that the CSR value of the thymoma group was $1.02 \pm 0.07$, the non-thymoma group was $0.64 \pm 0.10$. The difference was significant with $p<0.0001$. Our results suggest that CSR can be used as the sole criteria for discriminating between thymoma and non-thymoma. Inaoka T's study found that the CSR of the hyperplasia group was $0.614 \pm 0.13$, and the CSR of the thymoma group was $1.026 \pm 0.039(p<0.001)$ [15]. Priola AM identified the CSR value of the hyperplasia group was $0.545 \pm 0.162$, and of the thymoma group was $1.045 \pm 0.094[17]$.

\section{Logistic regression analysis}

Logistic regression analysis allows us to evaluate the probability of occurring an event when there are relevant factors. In our study, regression analysis determined the probability 


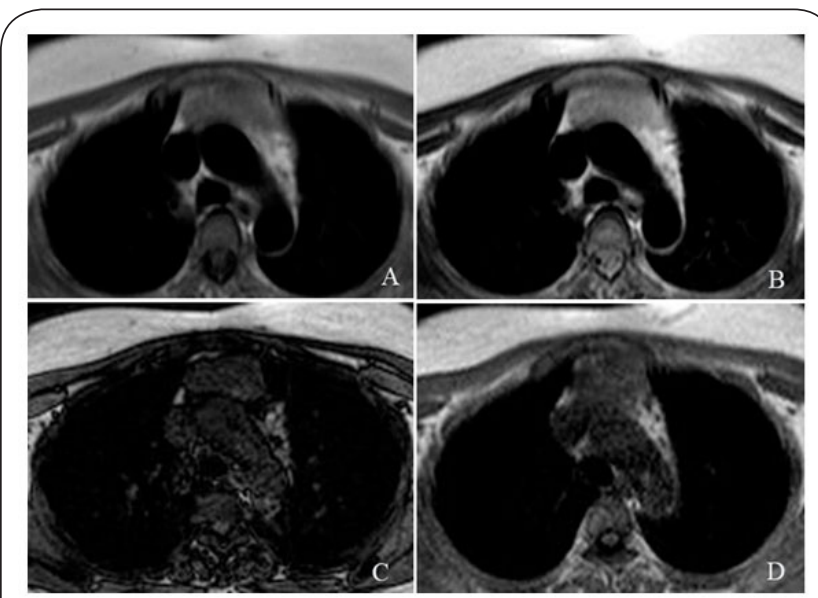

Figure 3. A 27-year-old woman with TLH. T1-weight (A) and T2-weight (B) showed an oval-shaped gross mass anterior to the aortic arch, which exhibits homogeneous soft-tissue signal intensity. In the opposed-phase image (C), mass showed the absence of signal intensity loss relative to the in-phase image (D). CSR value were 0.68 .

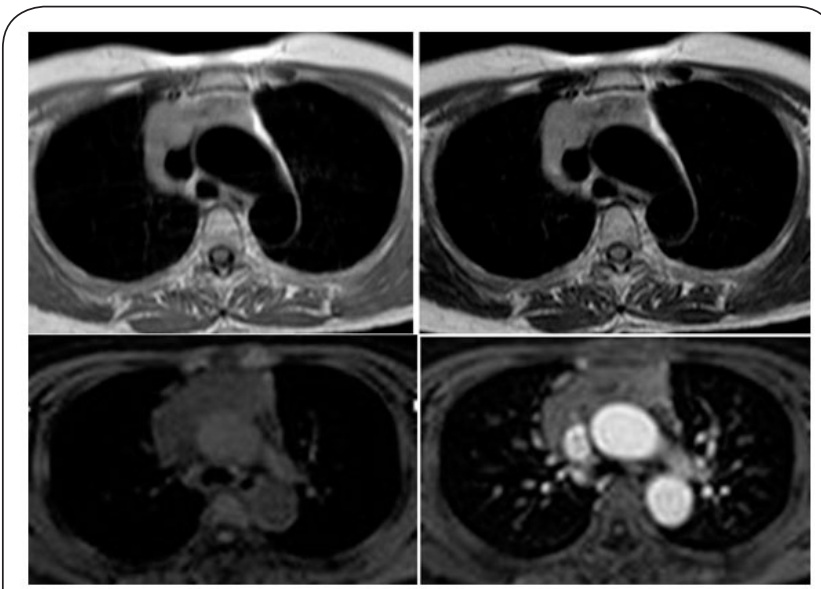

Figure 4. A 45-year-old woman with thymoma type B3.

TransverseT1-weighted and T2-weighted MR image shows a large mass withinvasion of SVC and innominate vein. The fat planes were obliterated completely. Contrast-enhanced MRI shows mass with homogeneous enhancement.

of occurrence of thymoma when there were distinct signals. The results in Table 3 showed that only CSR value were significantly different between thymoma and non-thymoma with $\mathrm{OR}=10.566$ and $\mathrm{p}=0.012$. Thus, with any location, shape and signal intensity, as the CSR value increases by 0.1 the likelihood of developing thymoma increases by about 10 times.

Although some studies about characteristic figures of thymoma and thymic hyperplasia have been published, now there are only two Pirola AM'studies assess the role of qualitative and quantitative factors MRlin predicting thymoma.In the logistic regression model for CSR, age and $\mathrm{BMI}$, odds ratio was 1.57 per $0.01 \mathrm{CSR}$ increase $(95 \% \mathrm{Cl}: 1.00,2.45)$ [17]. In the logistic regression model for qualitative parameters and SII, Priola AM found that SII were significantly correlatedwith the probability of finding thymoma, because the probability of tumor presence decreased with increased SII (OR per $1 \%$ increase, $0.64 ; 95 \% \mathrm{Cl}: 0.71-0.58$ ) [6]. Therefore, an increase 0.01 of value CSR or $1 \%$ of value SII for fixed age and gender leads to anincrease of 1.5 times and reduction of $36 \%$, respectively, in the probability of finding thymoma.

\section{Value of qualitative and quantitative analyses of MRI in thymoma diagnosis}

For qualitative analysis, the distinction between thymoma and non-thymoma was mainly based on observing the presence or absence of signal intensity loss on the opposed-phase image relative to the in-phase image. According to the results in Table 3, when using qualitative analyses to diagnose tumors, MRI had Se 97.3\%, Sp 88\%, Acc 93.6\%.

3 patients with hyperplasia misdiagnosis thymoma due to no appreciable signal intensity loss on the opposed-phase imaging relative to the in-phase imaging. These patients comprised 2 mass with CSR 0.68 and 0.7. One 22 year-old woman with gland and value of CSR was 1.0. One thymoma misdiagnosis hyperplasia because the mass was too small and embedded in the fatty infiltration gland.

For quantitative analysis, the CSR value was used as the sole criteria for distinguishing between thymoma and nonthymoma. The result in Figure 2 showed the AUROC value was 0.984 and CSR value was very good criteria for distinguishing tumors. The optimal cut-off of CSR value was 0.825 with Youden index 0.96, Se 100\% and Sp 96\%. Thus, quantitative analysis of MRI had Se, Sp higher than qualitative analysis and correctly diagnosed up to 61 cases. Quantitative assessment not only correctly diagnosed as well as qualitative assessment but also correctly diagnosed more 3 cases. The only one case misdiagnosed at quantitative assessment was a 22-year-old woman with hyperplasia but CSR value 1.0.

Combining both analyses, we used the CSR value of 0.825 as a criterion for discriminating thymoma from non-thymoma and re-diagnosed 4 false-positive cases in qualitative analysis. At that time, the two cases with soft tissue mass and no observed the signal intensity loss was re-diagnosed as hyperplasia due to low CSR values. A small tumor embedded in completely fatty gland was also re-diagnosed due to having a high CSR value. The results in Table 3 showed that combination of both quantitative and qualitative analyses, MRI had Se $100 \%$, Sp $96 \%$, Acc $98.4 \%$. This result was similar to the result of sole quantitative analysis.

According to Priola AM, for MRI, quantitative analysis applied alone or combined with qualitative analysis was more accurate than qualitative analysis applied alone. Combination of both qualitative and quantitative analyses, MRI had Se $100 \%$, Sp 98.5\%, Acc 98.8\% [6]. The studies of Inaoka T [15], Popa G [16] confirmed the use of CSR value as a sole criteria for discriminating thymomaMRI diagnosed accurately $100 \%$, 
Huy et al, Medical Imaging and Radiology 2019,

and there was no overlap in range.

\section{Limitations}

There are some limitations on our study.First, due to only patients underwent surgery were included, it is not consecutive and not representative for thymus imaging in patients with MG. Second, non dual-echo technique were performed in 5 patients. Because of the in-phase and opposed-phase images obtained in different times from two radiofrequency excitations, the misregistration could mislead the observation of signal intensity loss.

\section{Conclusion}

In patients with MG, due to a large number of thymic hyperplasia with mass form, the misdiagnosis could happen and cause unnecessary surgery. Chemical shift magnetic resonance imaging is helpful in differentiating thymoma from normal or hyperplastic thymus based on quantifying fat tissue.

\section{Competing interests}

The authors declare that they have no competing interests.

\section{Authors' contributions}

\begin{tabular}{|l|c|c|}
\hline Authors' contributions & HQH & PAT \\
\hline Research concept and design & $\checkmark$ & $\checkmark$ \\
\hline Collection and/or assembly of data & -- & $\checkmark$ \\
\hline Data analysis and interpretation & $\checkmark$ & $\checkmark$ \\
\hline Writing the article & $\checkmark$ & $\checkmark$ \\
\hline Critical revision of the article & $\checkmark$ & -- \\
\hline Final approval of article & $\checkmark$ & -- \\
\hline Statistical analysis & -- & $\checkmark$ \\
\hline
\end{tabular}

\section{Acknowledgments}

The authors are grateful to physicians, administrative staff at 103 Military Hospital and Cho Ray Hospitals for allowing us to undertake this research.

\section{Publication history}

Senior Editor: Domenico Rubello, Santa Maria della Misericordia Hospital, Italy.

Received: 31-Dec-2018 Final Revised: 28-Feb-2019

Accepted: 05-Mar-2019 Published: 29-Mar-2019

\section{References}

1. Onodera $\mathrm{H}$. The role of the thymus in the pathogenesis of myasthenia gravis. Tohoku J Exp Med. 2005; 207:87-98. | Article | PubMed

2. Ma YQ, Miao N, Abulajiang G, Li QX, Liu X, Zhang W, Wang CF and Wang J. [Clinicopathologic analysis of $\mathbf{5 2}$ cases of thymic epithelial tumor]. Zhonghua Bing Li Xue Za Zhi. 2010; 39:249-54. | Article | PubMed

3. Sadohara J, Fujimoto K, Muller NL, Kato S, Takamori S, Ohkuma K, Terasaki $\mathrm{H}$ and Hayabuchi $\mathrm{N}$. Thymic epithelial tumors: comparison of CT and MR imaging findings of low-risk thymomas, high-risk thymomas, and thymic carcinomas. Eur J Radiol. 2006; 60:70-9. | Article | PubMed

4. Marom EM, Rosado-de-Christenson ML, Bruzzi JF, Hara M, Sonett JR and Ketai L. Standard report terms for chest computed tomography reports of anterior mediastinal masses suspicious for thymoma. J Thorac Oncol. 2011; 6:S1717-23. | Article | PubMed
5. Pirronti T, Rinaldi P, Batocchi AP, Evoli A, Di Schino C and Marano P. Thymic lesions and myasthenia gravis. Diagnosis based on mediastinal imaging and pathological findings. Acta Radiol. 2002; 43:380-4. | PubMed

6. Priola AM, Priola SM, Gned D, Giraudo MT, Fornari A and Veltri A. Comparison of $\mathrm{CT}$ and chemical-shift MRI for differentiating thymoma from non-thymomatous conditions in myasthenia gravis: value of qualitative and quantitative assessment. Clin Radiol. 2016; 71:e157-69. | Article | PubMed

7. Marom EM. Imaging thymoma. J Thorac Oncol. 2010; 5:S296-303. Article I PubMed

8. Nasseri F and Eftekhari F. Clinical and radiologic review of the normal and abnormal thymus: pearls and pitfalls. Radiographics. 2010; 30:41328. | Article | PubMed

9. McErlean A, Huang J, Zabor EC, Moskowitz CS and Ginsberg MS. Distinguishing benign thymic lesions from early-stage thymic malignancies on computed tomography. J Thorac Oncol. 2013; 8:967-73. | Article | PubMed Abstract | PubMed FullText

10. Jeong YJ, Lee KS, Kim J, Shim YM, Han J and Kwon OJ. Does CT of thymic epithelial tumors enable us to differentiate histologic subtypes and predict prognosis? AJR Am J Roentgenol. 2004; 183:283-9. | Article | PubMed

11. Jung KJ, Lee KS, Han J, Kim J, Kim TS and Kim EA. Malignant thymic epithelial tumors: CT-pathologic correlation. AJR Am J Roentgenol. 2001; 176:433-9. | Article | PubMed

12. Baron RL, Lee JK, Sagel SS and Peterson RR. Computed tomography of the normal thymus. Radiology. 1982; 142:121-5. | Article | PubMed

13. G. popa, I. G. Lupescu and Georgescu1 S.A. The thymus in myasthenic patients: CT- pathologic correlation. ECR 2011/C-0751. 2011.

14. Takahashi K, Inaoka T, Murakami N, Hirota H, Iwata K, Nagasawa K, Yamada T, Mineta $\mathrm{M}$ and Aburano T. Characterization of the normal and hyperplastic thymus on chemical-shift MR imaging. AJR Am J Roentgenol. 2003; 180:1265-9. | Article | PubMed

15. Inaoka T, Takahashi K, Mineta M, Yamada T, Shuke N, Okizaki A, Nagasawa $\mathrm{K}$, Sugimori $\mathrm{H}$ and Aburano T. Thymic hyperplasia and thymus gland tumors: differentiation with chemical shift MR imaging. Radiology. 2007; 243:869-76. | Article | PubMed

16. Popa GA, Preda EM, Scheau C, Vilciu C and Lupescu IG. Updates in MRI characterization of the thymus in myasthenic patients. J Med Life. 2012; 5:206-10. | PubMed Abstract | PubMed FullText

17. Priola AM, Priola SM, Ciccone G, Evangelista A, Cataldi A, Gned D, Paze $F$, Ducco L, Moretti F, Brundu M and Veltri A. Differentiation of rebound and lymphoid thymic hyperplasia from anterior mediastinal tumors with dual-echo chemical-shift MR imaging in adulthood: reliability of the chemical-shift ratio and signal intensity index. Radiology. 2015; 274:238-49. | Article | PubMed

\section{Citation:}

Huy HQ and Tuan PA. Chemical-shift MRI for diagnosis thymoma in myasthenia gravis: Value of qualitative and quantitative assessment. Med Imaging Radiol. 2019; 7:2.

http://dx.doi.org/10.7243/2054-1945-7-2 